\title{
Fermion Zero Modes for Abelian BPS Monopoles
}

\author{
B. Cheng* and C. Ford ${ }^{\dagger}$ \\ Department of Mathematics \\ Imperial College London \\ London SW7 2AZ
}

June 16, 2021

\begin{abstract}
Fermion zero modes for abelian BPS monopoles are considered. In the spherically symmetric case the normalisable zero modes are determined for arbitrary monopole charge $N$. If $N>1$ the zero modes are zero along $N-1$ half-lines emanating from the monopole.
\end{abstract}

Keywords: abelian gauge theory, BPS monopoles, Weyl equation, fermion zero modes.

Fermion zero modes for BPS monopoles can be constructed via the same Nahm transform used to obtain the monopoles [1]. The construction is, however, cumbersome for magnetic charge $N>2$. In this letter we obtain zero modes for abelian BPS monopoles. Our approach is to directly integrate the Weyl equations in three-dimensional space rather than use Nahm's method (which has been adapted to abelian monopoles in [2]).

The abelian BPS equations read

$$
\mathbf{B}=\nabla \Phi
$$

where $\Phi$ is a real Higgs field and $\mathbf{B}$ is a magnetic field derived from a vector potential A. The Maxwell equation $\nabla \cdot \mathbf{B}=0$ implies that the Higgs field $\Phi$

*bobby.cheng11@imperial.ac.uk

${ }^{\dagger}$ c.ford@imperial.ac.uk 
obeys the Laplace equation. The Higgs field

$$
\Phi=\frac{g}{2 \pi}\left(a-\frac{1}{2} \sum_{i=1}^{N} \frac{1}{\left|\mathbf{r}-\mathbf{r}_{i}\right|}\right),
$$

with $a$ and $g$ constant, is harmonic away from $N$ singularities $\mathbf{r}_{i}(i=1,2, \ldots, N)$. Physically, the system comprises $N$ Dirac monopoles each with magnetic charge $g$ interacting with a Higgs field. Here $a$ fixes the asymptotic value of the Higgs field. Consider the Weyl operators

$$
D=e I_{2} \Phi+i \boldsymbol{\sigma} \cdot(-i \nabla+e \mathbf{A}) \quad D^{\dagger}=e I_{2} \Phi-i \boldsymbol{\sigma} \cdot(-i \nabla+e \mathbf{A})
$$

where $e$ is the electric charge of the fermion and $\boldsymbol{\sigma}=\left(\sigma_{1}, \sigma_{2}, \sigma_{3}\right)$. These Weyl operators assume a real Yukawa coupling in Minkowski space. However, identifying $\Phi$ as $A_{0}$ they are also Weyl operators for self-dual monopoles defined in Euclidean space.

The Dirac quantisation condition requires $e g=2 \pi n$ with $n$ integer. If

$$
e g=2 \pi
$$

and $a>0, D^{\dagger}$ has $N$ normalisable zero modes. In the $N=1$ case we have

$$
\Phi=\frac{g}{2 \pi}\left(a-\frac{1}{2 r}\right), \quad \mathbf{A}=\frac{g}{4 \pi} \frac{y \mathbf{e}_{x}-x \mathbf{e}_{y}}{r(r-z)}=-\frac{g(1+\cos \theta) \mathbf{e}_{\phi}}{4 \pi r \sin \theta},
$$

taking the origin as the location of the monopole and $(r, \theta, \phi)$ denote spherical polar coordinates. Here the Dirac string lies on the positive $z$-axis.

One can verify that

$$
\psi=\frac{e^{-a r}}{\sqrt{r}}\left(\begin{array}{c}
-\sin (\theta / 2) \\
\cos (\theta / 2) e^{i \phi}
\end{array}\right)
$$

is a zero mode of $D^{\dagger}$ (the components of $D^{\dagger}$ in spherical polar coordinates are given in the appendix). $D$ has no zero modes. This result can be obtained by taking the large $r$ limit of the Jackiw-Rebbi zero mode [3, 4] for the basic $S U(2)$ BPS monopole after performing a gauge transformation which diagonalises the Higgs field. The fermion density $\psi^{\dagger} \psi=e^{-2 a r} / r$ is spherically symmetric and has an integrable singularity at the monopole centre.

The general $N$ case is more complicated. However, in the spherically symmetric case where the positions of the $N$ monopoles coincide the Higgs field is

$$
\Phi=\frac{g}{2 \pi}\left(a-\frac{N}{2 r}\right)
$$


and $\mathbf{A}$ is the $N=1$ potential multiplied by $N$. Here $D^{\dagger}$ has $N$ normalisable zero modes:

$\psi^{m}=r^{\frac{1}{2}(N-2)} e^{-a r}\left(\begin{array}{c}-\sin ^{N-m+1}(\theta / 2) \cos ^{m-1}(\theta / 2) e^{i(m-1) \phi} \\ \sin ^{N-m}(\theta / 2) \cos ^{m}(\theta / 2) e^{i m \phi}\end{array}\right) \quad m=1,2, \ldots, N$.

These solutions resemble (in particular with respect to the $\theta$ and $\phi$ dependence) known solutions of the Dirac equation in the background of abelian monopoles [5]. However, our solutions are written directly in terms of trigonometric functions rather than spherical harmonic: 1 . Our solutions are normalisable with $L^{2}$ norm $4 \pi(N-m) !(m-1) !(2 a)^{-(N+1)}$. As the zero modes $\psi^{m}$ are annihilated by the Hamiltonian it is not clear to us whether the presence of the Higgs field cures the self-adjointness problem [5, 7] associated with monopole Hamiltonians. To address this issue one needs to study the scattering states [5].

Note that the densities $\psi^{m \dagger} \psi^{m}$ are not spherically symmetric for $N>1$. For $N=2$ the first zero mode is zero along the positive $z$-axis while the second mode is zero on the negative $z$-axis. By taking a suitable linear combination of $\psi^{1}$ and $\psi^{2}$ one can obtain a zero mode with a zero along any half-line emanating from the monopole; the zero mode is axially symmetric about the axis on which the half-line lies. The $N>1$ zero modes are zero along $N-1$ half-lines. Our $\psi^{1}$ and $\psi^{N}$ have zeros of strength $N-1$ on the positive and negative $z$-axes, respectively; the remaining $N-2$ modes have lower strength zeros on both the positive and negative $z$ axes. Again one can adjust the directions of the $N-1$ half lines by taking different linear combinations of the $N$ zero modes. For a discussion of zeros of fermion zero modes in a different context see [8].

In general, the $N>2$ zero modes are not axially symmetric even though the $\psi^{m}$ are all axially symmetric about the $z$-axis. If $\Psi$ is a linear combination of the $\psi^{m}$, the density $\Psi^{\dagger} \Psi$ has the form

$$
\Psi^{\dagger} \Psi=r^{N-2} e^{-2 a r} f(\theta, \phi)
$$

where $f$ is a function of $\theta$ and $\phi$. For $N>2$ one can see that the zero modes vanish at the position of the monopole and the zero modes peak somewhere on the sphere $r=(N-2) / 2 a$. The function $f(\theta, \phi)$ has up to $N-1$ zeros; if there are fewer than $N-1$ zeros these are repeated zeros associated with coincident half-lines. Examination of $f(\theta, \phi)$ for several zero modes indicates that $f(\theta, \phi)$ has a single peak. For example, the $N=3$

\footnotetext{
${ }^{1}$ If $N$ is even the zero modes can be expressed in terms of standard spherical harmonics. If $N$ is odd spin-weighted or monopole harmonics [6] are required.
} 

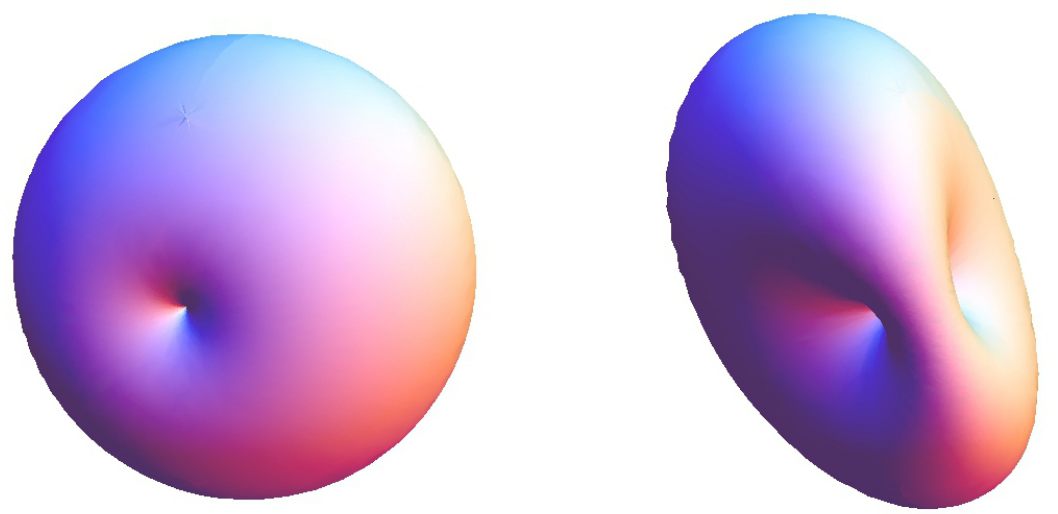

Figure 1: Spherical plots of $f(\theta, \phi)$ for an $N=2$ and $N=3$ zero mode (the scale depends on the normalisation and hence $a)$. The left plot shows $f(\theta, \phi)$ for an $N=2$ zero mode $\left(\Psi=\psi^{1}+\psi^{2}\right)$ which has one zero and is axially symmetric. Two zeros are visible for the $N=3$ zero mode $\left(\Psi=\psi^{1}+\psi^{2}-i \psi^{3}\right)$ on the right. Both zero modes have a single peak.

zero mode $\Psi=\psi^{1}+\psi^{2}+\psi^{3}$ yields an $f(\theta, \phi)$ function with two zeros and a maximum on the equator $\theta=\pi / 2$ (these three points are equally spaced on the equator). The maximum of $f(\theta, \phi)$ is a point on the unit sphere except for some axially symmetric solutions where $f(\theta, \phi)$ peaks on a circle. In Figure 1 spherical plots of $f(\theta, \phi)$ are given for an $N=2$ and an $N=3$ zero mode.

van Baal [9] has given an ansatz which provides a solution of the Weyl equation for any solution of the abelian BPS equation:

$$
\psi_{v B}=D\left(\begin{array}{l}
w \\
0
\end{array}\right)
$$

satisfies $D^{\dagger} \psi_{v B}=0$ where

$$
\Phi=\frac{g}{2 \pi} \frac{\partial}{\partial z} \log w, \quad \mathbf{A}=-\frac{g}{2 \pi} \mathbf{k} \times \nabla \log w
$$

and $\log w$ satisfies the Laplace equation. This works as the BPS equation implies $D^{\dagger} D$ is a scalar and 10 gives $D^{\dagger} D w=0$. Taking $\log w=\frac{1}{2} N \log (r-z)+a z$ yields our $\Phi$ and $\mathbf{A}$. However, $\psi_{v B}$ is not normalisable ${ }^{2}$ though for $a=0$ it agrees with our zero mode $\psi^{1}$. Indeed, $\psi^{1}$ is the

\footnotetext{
${ }^{2}$ Remarkably, (9) does provide one normalisable zero mode for a different class of Higgs fields; here $\Phi$ has $2 N$ singularities representing $N$ positively charged and $N$ negatively charged monopoles [9, 10].
} 
$a=0$ van Baal solution multiplied by $e^{-a r}$. As the van Baal construction does not rely on spherical symmetry this approach may provide information about the $a=0$ limit of the general case where the $N$ monopoles are separated.

We have considered fermion zero modes for BPS monopoles and have obtained solutions for arbitrary magnetic charge $N$. The spherically symmetric abelian case we have solved may provide a model for non-abelian magnetic bags; although higher charge $S U(2)$ BPS monopoles are never spherically symmetric, solutions with approximate spherical symmetry may emerge for large $N$ [11]. It would be interesting to investigate the extent to which our zero modes approximate the fermion zero modes of magnetic bags.

\section{Appendix}

The components of $D^{\dagger}$ associated with (6) are $(e g=2 \pi)$

$$
\begin{aligned}
& \left(D^{\dagger}\right)_{11}=a-\frac{N}{2 r}-\cos \theta \frac{\partial}{\partial r}+\frac{\sin \theta}{r} \frac{\partial}{\partial \theta} \\
& \left(D^{\dagger}\right)_{12}=e^{-i \phi}\left[-\sin \theta \frac{\partial}{\partial r}-\frac{\cos \theta}{r} \frac{\partial}{\partial \theta}+\frac{i}{r \sin \theta} \frac{\partial}{\partial \phi}+\frac{N}{2} \frac{(1+\cos \theta)}{r \sin \theta}\right] \\
& \left(D^{\dagger}\right)_{21}=e^{i \phi}\left[-\sin \theta \frac{\partial}{\partial r}-\frac{\cos \theta}{r} \frac{\partial}{\partial \theta}-\frac{i}{r \sin \theta} \frac{\partial}{\partial \phi}-\frac{N}{2} \frac{(1+\cos \theta)}{r \sin \theta}\right] \\
& \left(D^{\dagger}\right)_{22}=a-\frac{N}{2 r}+\cos \theta \frac{\partial}{\partial r}-\frac{\sin \theta}{r} \frac{\partial}{\partial \theta} .
\end{aligned}
$$

\section{References}

[1] W. Nahm, Phys. Lett. B 90 (1980) 413.

[2] W. Nahm, Phys. Lett. B 93 (1980) 42.

[3] R. Jackiw and C. Rebbi, Phys. Rev. D 13 (1976) 3398.

[4] A. Gonzalez-Arroyo and Y. A. Simonov, Nucl. Phys. B 460 (1996) 429 hep-th/9506032.

[5] Y. Kazama, C. N. Yang and A. S. Goldhaber, Phys. Rev. D 15 (1977) 2287.

[6] T. T. Wu and C. N. Yang, Nucl. Phys. B 107 (1976) 365. 
[7] H. J. Lipkin, W. I. Weisberger and M. Peshkin, Annals Phys. 53 (1969) 203.

[8] F. Bruckmann, Phys. Rev. D 71 (2005) 101701 hep-th/0411252].

[9] P. van Baal, in: Confinement, Topology, and other Non-Perturbative Aspects of QCD, eds. J. Greensite and S. Olejnik, NATO Science Series, Vol. 83 (Kluwer, Dordrecht, 2002) [hep-th/0202182].

[10] F. Bruckmann, D. Nogradi and P. van Baal, Nucl. Phys. B 666 (2003) 197 hep-th/0305063].

[11] S. Bolognesi, Nucl. Phys. B 752 (2006) 93 hep-th/0512133. 